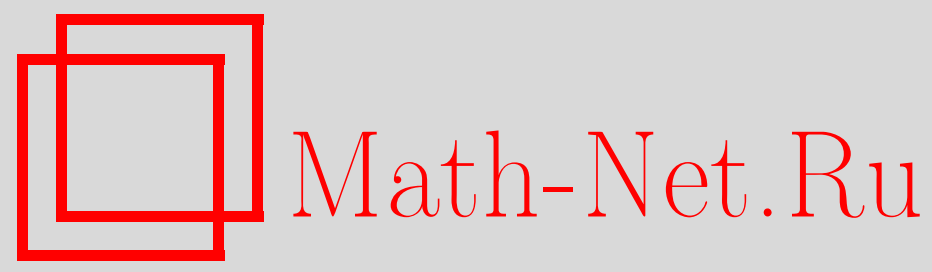

А. В. Чуркин, Стохастическое уравнение Шрёдингера для квантового осциллятора с учетом диссипации, Матем. заметки, 2005, том 78, выпуск 6, 934-940

DOI: https://doi.org/10.4213/mzm2652

Использование Общероссийского математического портала Math-Net.Ru подразумевает, что вы прочитали и согласны с пользовательским соглашением http://www . mathnet.ru/rus/agreement

Параметры загрузки:

IP: 18.208 .226 .222

26 апреля 2023 г., 13:49:55 


\title{
СТОХАСТИЧЕСКОЕ УРАВНЕНИЕ ШРЁДИНГЕРА ДЛЯ КВАНТОВОГО ОСЦИЛЛЯТОРА С УЧЕТОМ ДИССИПАЦИИ
}

\author{
А. В. Чуркин
}

\begin{abstract}
В работе построено точное решение стохастического уравнения Шрёдингера для квантового осциллятора с учетом возможной диссипации энергии. Используя полученный явный вид решения, удалось вычислить оценки характерного времени затухания для свободных затухающих колебаний. В случае вынужденных колебаний получена формула для добротности системы, а также для дисперсии координаты и импульса квантового осциллятора с учетом диссипации. Получен квантовый аналог классического уравнения диффузии и показано в явном виде, что уравнения движения для среднего значения оператора импульса, следуюшие из решения стохастического уравнения Шрёдингера, играют роль квантового уравнения Ланжевена, описьвающего броуновское движение под действием стохастической силы.
\end{abstract}

Библиографория: 10 названий.

Введение. Одной из самых широко известных и часто используемых моделей в квантовой механике является квантовьй осциллятор. С одной стороны, математическая задача Коши для уравнения Шрёдингера с квадратичным гамильтонианом свободного гармонического осциллятора решается точно в явном виде, с другой стороны, модель квантового осциллятора описьвает поведение весьма разнообразных квантовомеханических объектов. В классической физике теория затухающих гармонических колебаний является прямым обобщением теории свободных незатухающих колебаний. Как известно, она решается путем добавления к уравнению движения диссипативного слагаемого.

В квантовой физике задача о колебаниях с учетом диссипации сводится к рассмотрению вопроса о взаимодействии квантовой системы с окружением. За последние несколько десятков лет было опубликовано большое число работ, посвященных различным подходам к описанию этой проблемы (см., например, [1]-[5]). Так, например, в работах [6], [7] для описания эволюции во времени квантового осциллятора с учетом диссипации используется марковское управляющее уравнение как обобщение уравнения Лиувилля на случай открытой квантовой системы.

В данной статье рассматривается другой подход к описанию открытой квантовой системы: эволюция во времени квантового осциллятора с диссипацией задается решением стохастического уравнения Шрёдингера.

Работа выполнена при поддержке Российского фонда фундаментальных исследований, грант № 05-01-00824. 
1. Решение стохастического уравнения Шрёдингера для осциллятора с учетом диссипации. Стохастическое уравнение Шрёдингера было предложено в работах [2], [8], [9] для описания процесса квантового непрерьвного измерения в следующем виде:

$$
d \Psi(t)=\left(-\frac{i}{\hbar} \widehat{H}-\frac{\lambda}{4}(\widehat{A}-a(t))^{2}\right) \Psi(t) d t+\sqrt{\frac{\lambda}{2}}(\widehat{A}-a(t)) \Psi(t) d Q(t) .
$$

Здесь $\widehat{H}$ - гамильтониан системы, $\widehat{A}$ - оператор некоторой наблюдаемой, $a(t)=(\Psi, \widehat{A} \Psi)$ - среднее значение наблюдаемой $\widehat{A}, \lambda$ - константа, характеризующая интенсивность взаимодействия между измеряемой квантовой системой и измерительным прибором, $d Q(t)$ - дифференциал стандартного винеровского случайного процесса.

Воспользуемся уравнением (1) для описания эволюции во времени квантового осциллятора с учетом диссипации. Для этого в качестве оператора наблюдаемой $A$ выберем оператор уничтожения $\hat{b}$, которьй в координатном представлении имеет вид

$$
\widehat{A}=x+\gamma \frac{d}{d x}
$$

Рассмотрим гамильтониан квантового осциллятора, находящегося под действием некоторой внешней периодической силы $f(t)$ :

$$
\widehat{H}=-\frac{\hbar^{2}}{2 M} \frac{\partial^{2}}{\partial x^{2}}+\frac{M \omega_{0}^{2}}{2} x^{2}+f(t)
$$

где $f(t)=f_{0} \cos (\omega t), M$ и $\omega_{0}$ - масса и собственная частота осциллятора, $f_{0}$ и $\omega-$ aмплитуда и частота вынуждающей силы. Стохастическое уравнение Шрёдингера (1) с наблюдаемой (2) в координатном представлении примет вид

$$
\begin{aligned}
d \Psi(t)= & -\frac{i}{\hbar} \widehat{H} \Psi(t) d t-\frac{\lambda}{4}\left(\hat{x}-q(t)+\gamma \frac{d}{d x}\right)\left(\hat{x}-q(t)-\gamma \frac{d}{d x}\right) \Psi(t) d t \\
& +\sqrt{\frac{\lambda}{2}}\left(\hat{x}-q(t)+\gamma \frac{d}{d x}\right) \Psi(t) d Q(t) .
\end{aligned}
$$

Группируя вместе слагаемые, содержащие параметр $\gamma=0$, получаем

$$
\begin{aligned}
d \Psi(t)= & -\frac{i}{\hbar} \widehat{H} \Psi(t) d t-\frac{\lambda}{4}(\hat{x}-q(t))^{2} \Psi(t) d t+\sqrt{\frac{\lambda}{2}}(\hat{x}-q(t)) \Psi(t) d Q(t) \\
& +\gamma\left(\frac{\lambda}{4}\left(\gamma \frac{d^{2}}{d x^{2}}+1\right) \Psi(t) d t+\sqrt{\frac{\lambda}{2}} \frac{d}{d x} \Psi(t) d Q(t)\right)
\end{aligned}
$$

Если $\gamma=0$, решение уравнения (4) имеет вид (подробное решение для данного случая изложено в статье [10])

$$
\Psi_{0}(x, t)=N_{0}(t) \exp \left(R_{0}(t)\left(x-q_{0}(t)\right)^{2}+\frac{i}{\hbar} x p_{0}(t)\right),
$$


где $N(t)$ - нормировочная константа, $R_{0}(t)$ - решение следующего уравнения Риккати:

$$
\frac{d R_{0}}{d t}=\frac{2 i \hbar}{M} R_{0}^{2}-\frac{i M \omega_{0}^{2}}{2 \hbar}-\frac{\lambda}{2}
$$

a переменные $q_{0}(t)$ и $p_{0}(t)$ являются решением стохастической гамильтоновой системы

$$
\begin{aligned}
& d q_{0}=\frac{p_{0}(t)}{M} d t-\frac{1}{2 \operatorname{Re}\left(R_{0}(t)\right)} \sqrt{\frac{\lambda}{2}} d Q, \\
& d p_{0}=-\left(M \omega_{0}^{2} q_{0}(t)+f_{0} \cos (\omega t)\right) d t-\hbar \frac{\operatorname{Im}\left(R_{0}(t)\right)}{\operatorname{Re}\left(R_{0}(t)\right)} \sqrt{\frac{\lambda}{2}} d Q .
\end{aligned}
$$

Согласно [10] решение (5)-(8) описьвает процедуру квантового непрерьвного измерения координаты осциллятора. Рассмотрим теперь решение уравнения (4) с $\gamma \neq 0$. В этом случае обший вид решения (5) не изменится:

$$
\Psi(x, t)=N(t) \exp \left(R(t)(x-q(t))^{2}+\frac{i}{\hbar} x p(t)\right)
$$

но уравнения для коэффициентов будут более сложными. Так функция $R(t)$, определяющая обратную величину дисперсии решения (9), удовлетворяет следующему уравнению Риккати:

$$
\frac{d R}{d t}=\frac{2 i \hbar}{M} R^{2}-\frac{i M \omega_{0}^{2}}{2 \hbar}-\lambda\left(\frac{1}{2}+\gamma R\right) .
$$

Решение данного уравнения может быть сформулировано в виде следующей теоремы.

Теорема. Решение уравнения (10) имеет вид

$$
R(t)=-b+\frac{\sqrt{c / a} \operatorname{tg}(\sqrt{a c} t)+b+R(0)}{1-\sqrt{a / c}(b+R(0)) \operatorname{tg}(\sqrt{a c} t)},
$$

$2 \partial e$

$$
a=\frac{2 i \hbar}{M}, \quad b=i \frac{\gamma \lambda M}{4 \hbar}, \quad c=-\frac{i M \omega_{0}^{2}}{2 \hbar}-\frac{\lambda}{2}+i \frac{\gamma^{2} \lambda^{2} M}{8 \hbar},
$$

при этом при больших $t$ имеет место следующая асимптотика:

$$
\lim _{t \rightarrow \infty}(R(t))= \begin{cases}-\frac{M \omega_{0}}{2 \hbar} & n p u \gamma=-\frac{\hbar}{M \omega_{0}} \\ -\frac{M \omega_{0}}{2 \hbar} \sqrt{1-i \frac{\hbar \lambda}{M \omega_{0}^{2}}} & n p u \gamma=0, \\ -i \frac{\lambda}{4 \omega_{0}}-\sqrt{\left(\frac{M \omega_{0}}{2 \hbar}+i \frac{\lambda}{4 \omega_{0}}\right)^{2}+i \frac{\lambda M}{2 \hbar}} & n p u \gamma=\frac{\hbar}{M \omega_{0}}\end{cases}
$$


ДокАЗАТЕЛьСТво. Для доказательства теоремы приведем уравнение (10) к следующей форме:

$$
\begin{aligned}
\frac{d R}{d t} & =\frac{2 i \hbar}{M}\left(R+i \frac{\gamma \lambda M}{4 \hbar}\right)^{2}-\frac{i M \omega_{0}^{2}}{2 \hbar}-\frac{\lambda}{2}+i \frac{\gamma^{2} \lambda^{2} M}{8 \hbar} \\
& =\frac{2 i \hbar}{M}\left(R+i \frac{\gamma \lambda M}{4 \hbar}\right)^{2}-\frac{2 i \hbar}{M}\left(\left(\frac{M \omega_{0}}{2 \hbar}+i \frac{\gamma \lambda M}{4 \hbar}\right)^{2}+i \frac{\lambda M}{4 \hbar}\left(1+\frac{M \omega_{0} \gamma}{\hbar}\right)\right)
\end{aligned}
$$

Решение полученного уравнения Риккати с комплексными коэффициентами может быть получено как обобщение хорошо известного уравнения Риккати с вещественными коэффициентами и записано в виде (11).

При больших временах $t$ решение (11) уравнения (10) при условии $\operatorname{Re}(R(t))<0$ стабилизируется. Следовательно,

$$
\lim _{t \rightarrow \infty} R(t)=-i \frac{\gamma \lambda M}{4 \hbar} \pm \sqrt{\left(\frac{M \omega_{0}}{2 \hbar}+i \frac{\gamma \lambda M}{4 \hbar}\right)^{2}+i \frac{\lambda M}{4 \hbar}\left(1+\frac{M \omega_{0} \gamma}{\hbar}\right)}
$$

Используя условие стабилизации $\operatorname{Re}(R(t))<0$, нетрудно вычислить асимптотические пределы (12) для различных значений параметра $\gamma$. Значения параметра $\gamma$ определяются выбором исходного оператора наблюдаемой $(2)$. Если $\gamma=-\hbar /\left(M \omega_{0}\right)$, то оператор наблюдаемой (2) пропорционален оператору уничтожения:

$$
\widehat{A}=\hat{b}=\sqrt{\frac{M \omega_{0}}{2 \hbar}}\left(x-\frac{\hbar}{M \omega_{0}} \frac{d}{d x}\right) .
$$

Если $\gamma=0$, то оператор наблюдаемой (2) пропорционален оператору координаты осциллятора:

$$
\widehat{A}=\sqrt{\frac{M \omega_{0}}{2 \hbar}} \hat{x} .
$$

Если $\gamma=\hbar /\left(M \omega_{0}\right)$, то оператор наблюдаемой $(2)$ пропорционален оператору рождения:

$$
\widehat{A}=\hat{b}^{+}=\sqrt{\frac{M \omega_{0}}{2 \hbar}}\left(x+\frac{\hbar}{M \omega_{0}} \frac{d}{d x}\right) .
$$

Подставляя решение (9) в исходное уравнение (4), получаем систему уравнений для коэффициентов квадратного полинома. Уравнение для коэффициентов при $x^{2} d t$ дает уравнение Риккати (10), уравнения для коэффициентов при $x d t$ и $x d Q$ ведут к следующей системе стохастических дифференциальных уравнений:

$$
\begin{aligned}
d q= & p(t)\left(\frac{1}{M}+\gamma^{2} \lambda \frac{\operatorname{Im}(R(t))}{2 \hbar \operatorname{Re}(R(t))}\right) d t-\left(\frac{1}{2 \operatorname{Re}(R(t))}+\gamma\right) \sqrt{\frac{\lambda}{2}} d Q \\
d p= & p(t) \gamma \lambda\left(\frac{1}{2}+\gamma\left(\operatorname{Re}(R(t))+\frac{\operatorname{Im}(R(t))^{2}}{\operatorname{Re}(R(t))}\right)\right) d t-\left(M \omega_{0}^{2} q(t)+f_{0} \cos (\omega t)\right) d t \\
& -\hbar \frac{\operatorname{Im}(R(t))}{\operatorname{Re}(R(t))} \sqrt{\frac{\lambda}{2}} d Q .
\end{aligned}
$$


Если $\gamma=0$, система $(14),(15)$ переходит в систему (7), (8). В случае $\gamma=-\hbar /\left(M \omega_{0}\right)$ после подстановки асимптотики $R(t)$ из (12) получается следующая система стохастических уравнений Гамильтона:

$$
\begin{aligned}
& d q=\frac{p(t)}{M} d t+\frac{2 M \omega_{0}}{\hbar} \sqrt{\frac{\lambda}{2}} d Q, \\
& d p=-p(t) \frac{\hbar \lambda}{M \omega_{0}} d t-\left(M \omega_{0}^{2} q(t)+f_{0} \cos (\omega t)\right) d t .
\end{aligned}
$$

Таким образом, если оператор наблюдаемой (2) пропорционален оператору уничтожения

$$
\widehat{A}=\hat{b}=\sqrt{\frac{M \omega_{0}}{2 \hbar}}\left(x-\frac{\hbar}{M \omega_{0}} \frac{d}{d x}\right),
$$

тог да коэффициенты $q(t)$ и $p(t)$ являются решением следуюшей системы стохастических дифференциальных уравнений:

$$
\begin{aligned}
& d q=\frac{p(t)}{M} d t+\kappa d Q, \\
& d p=-2 \nu p(t) d t-M \omega_{0}^{2} q(t) d t-f(t) d t,
\end{aligned}
$$

где

$$
\nu=\frac{\hbar \lambda}{2 M \omega_{0}}, \quad f(t)=M f_{0} \cos (\omega \tau), \quad \kappa=2 \frac{\hbar}{M \omega_{0}} \sqrt{\frac{\lambda}{2}} .
$$

Здесь $\nu$ - коэффициент затухания, $f(t)$ - регулярная внешняя сила и $\kappa$ - амплитуда стохастического процесса.

2. Вычисление среднего значения и дисперсии осциллятора. Рассмотрим решение системы $(16),(17)$ при наличии нулевых начальных условий: $q(0)=0, p(0)=0$. Ввиду линейности системы (16), (17), ее общее решение есть сумма общего решения однородной системы и частного решения неоднородной системы. По формуле Дюамеля общее решение однородной системы $(16),(17)$ имеет вид

$$
\left(\begin{array}{c}
q(t) \\
p(t)
\end{array}\right)=U_{t}\left(\begin{array}{c}
q(0) \\
p(0)
\end{array}\right)+\int_{0}^{t} d \tau U_{t-\tau}\left(\begin{array}{c}
0 \\
-f(\tau)
\end{array}\right)+\int_{0}^{t} d Q(\tau) U_{t-\tau}\left(\begin{array}{c}
\kappa \\
0
\end{array}\right)
$$

где

$$
U_{t}=e^{-\nu t}\left(\begin{array}{cc}
\cos (\Omega t)+\frac{\nu}{\Omega} \sin (\Omega t) & \frac{1}{M \Omega} \sin (\Omega t) \\
-M\left(\frac{\nu^{2}}{\Omega}+\Omega\right) \sin (\Omega t) & \cos (\Omega t)-\frac{\nu}{\Omega} \sin (\Omega t)
\end{array}\right), \quad \Omega=\sqrt{\omega_{0}^{2}-\nu^{2}} .
$$

При нулевых начальных условиях $q(0)=0, p(0)=0$ решение системы $(16),(17)$ состоит из регулярной части $\left(q_{c}(t)\right)$ и стохастической части $\left(q_{Q}(t)\right)$ :

$$
\begin{aligned}
q(t)=q_{c}(t)+q_{Q}(t)= & -\int_{0}^{t} f(\tau) \frac{\sin \Omega(t-\tau)}{M \Omega} e^{-\nu(t-\tau)} d \tau \\
& +\kappa \int_{0}^{t}\left(\cos \Omega(t-\tau)+\frac{\nu \sin \Omega(t-\tau)}{\Omega}\right) e^{-\nu(t-\tau)} d Q(\tau), \\
p(t)=p_{c}(t)+p_{Q}(t)= & -\int_{0}^{t} f(\tau)\left(\cos \Omega(t-\tau)-\frac{\nu \sin \Omega(t-\tau)}{\Omega}\right) e^{-\nu(t-\tau)} d \tau \\
& -\kappa M\left(\frac{\nu^{2}}{\Omega}+\Omega\right) \int_{0}^{t} \sin (\Omega t) e^{-\nu(t-\tau)} d Q(\tau) .
\end{aligned}
$$


Регулярная часть решения (18), (19) имеет классический вид

$$
\begin{aligned}
q_{c}(t)= & -\frac{f_{0} / M}{\left(\omega_{0}^{2}-\omega^{2}\right)^{2}+4 \nu^{2} \omega^{2}}\left(\left(\omega^{2}-\omega_{0}^{2}\right) \cos (\omega t)-2 \nu \omega \sin (\omega t)\right. \\
& \left.+e^{-\nu t}\left(\frac{\nu\left(\omega_{0}^{2}+\omega^{2}\right)}{\sqrt{\omega_{0}^{2}-\nu^{2}}} \sin \left(\sqrt{\omega_{0}^{2}-\nu^{2}} t\right)-\left(\omega^{2}-\omega_{0}^{2}\right) \cos \left(\sqrt{\omega_{0}^{2}-\nu^{2}} t\right)\right)\right), \\
p_{c}(t)=- & \frac{f_{0}}{\left(\omega_{0}^{2}-\omega^{2}\right)^{2}+4 \nu^{2} \omega^{2}}\left(\omega\left(\omega_{0}^{2}-\omega^{2}\right) \sin (\omega t)-2 \nu \omega^{2} \cos (\omega t)\right. \\
& \left.+e^{-\nu t}\left(\frac{\left(w_{0}^{4}+2 \nu^{2} \omega^{2}-\omega_{0}^{2} \omega^{2}\right)}{\sqrt{w_{0}^{2}-\nu^{2}}} \sin \left(\sqrt{\omega_{0}^{2}-\nu^{2}} t\right)-2 \nu \omega^{2} \cos \left(\sqrt{\omega_{0}^{2}-\nu^{2}} t\right)\right)\right) .
\end{aligned}
$$

В резонансном случае $\left(\omega_{0}=\omega\right)$ :

$$
\begin{aligned}
& q_{c}(t)=\frac{\left(f_{0} / M\right) \sin (\omega t)}{2 \nu \omega}-\frac{\left(f_{0} / M\right) e^{-\nu t} \sin \left(\sqrt{\omega_{0}^{2}-\nu^{2}} t\right)}{2 \nu \sqrt{\omega_{0}^{2}-\nu^{2}}} \\
& p_{c}(t)=\frac{f_{0} \cos (\omega t)}{2 \nu}-\frac{f_{0} e^{-\nu t}}{2 \nu}\left(\frac{\nu}{\sqrt{w_{0}^{2}-\nu^{2}}} \sin \left(\sqrt{\omega_{0}^{2}-\nu^{2}} t\right)-\cos \left(\sqrt{\omega_{0}^{2}-\nu^{2}} t\right)\right) .
\end{aligned}
$$

После затухания переходного процесса $(t \gg 1 / \nu)$ в резонансном случае получаем классическую часть решения в следующем виде:

$$
\begin{aligned}
& q_{c}(t) \rightarrow \frac{\left(f_{0} / M\right) \sin (\omega t)}{2 \nu \omega}=\frac{f_{0}}{\hbar \lambda} \sin (\omega t), \\
& p_{c}(t) \rightarrow \frac{f_{0} \cos (\omega t)}{2 \nu}=\frac{f_{0} M \omega}{\hbar \lambda} \cos (\omega t) .
\end{aligned}
$$

Для изучения влияния квантовых поправок к классической картине вычислим дисперсию стохастической части полученного решения (19):

$$
\begin{aligned}
& \left\langle(\Delta p(t))^{2}\right\rangle=\left\langle\left(p(t)-p_{c}(t)\right)^{2}\right\rangle=\kappa^{2} M^{2}\left(\frac{\nu^{2}}{\Omega}+\Omega\right)^{2} \int_{0}^{t} \sin ^{2}(\Omega t) e^{-2 \nu(t-\tau)} d \tau \\
& =\kappa^{2} M^{2}\left(\nu^{2}+\Omega^{2}\right)\left(\frac{\Omega^{2}+e^{-2 \nu t}\left(\nu^{2} \cos (2 \Omega t)-\nu \Omega \sin (2 \Omega t)-\left(\nu^{2}+\Omega^{2}\right)\right)}{4 \nu \Omega^{2}}\right) \\
& =\frac{\kappa^{2} M^{2} \omega_{0}^{2}}{4 \nu}\left(1+\frac{e^{-2 \nu t}\left(\nu^{2} \cos \left(2 \sqrt{\omega_{0}^{2}-\nu^{2}} t\right)-\nu \sqrt{\omega_{0}^{2}-\nu^{2}} \sin \left(2 \sqrt{\omega_{0}^{2}-\nu^{2}} t\right)-\omega_{0}^{2}\right)}{\omega_{0}^{2}-\nu^{2}}\right) .
\end{aligned}
$$

Если затухание происходит достаточно медленно по сравнению с ритмом собственных колебаний осциллятора $\left(\nu \ll \omega_{0}\right)$, то после затухания переходного процесса получаем $(t \gg 1 / \nu)$ :

$$
\left\langle(\Delta p(t))^{2}\right\rangle \approx \frac{\kappa^{2} M^{2} \omega_{0}^{2}}{4 \nu}\left(1-e^{-2 \nu t}\right) .
$$

Следовательно, при больших $t$ дисперсия $\left\langle\left(p(t)-p_{c}(t)\right)^{2}\right\rangle$ удовлетворяет классическому уравнению для броуновского движения

$$
\left\langle(\Delta p(t))^{2}\right\rangle=D t
$$


причем коэффищиент диффузии $D$ имеет вид

$$
D=\frac{\kappa^{2} M^{2} \omega_{0}^{2}}{2}=\lambda \hbar^{2} .
$$

В результате мы получили, что среднее значение оператора момента для квантового осциллятора с учетом диссипации удовлетворяет классическому уравнению Ланжевена, описьвающему броуновское движение частищы в вязкой среде под действием случайной силы:

$$
\dot{p}+\Gamma p=F(t)
$$

Здесь $\Gamma=\hbar \lambda /\left(M \omega_{0}\right)$ - коэффициент затухания Ланжевена, а сила $F(t)$ складывается из регулярной внешней силы $f(t)$ и стохастической силы $M \omega_{0} q(t)$.

Таким образом, выбор оператора уничтожения в качестве оператора наблюдаемой в стохастическом уравнении Шрёдингера (1) позволяют учесть диссипацию энергии квантового осциллятора. При этом среднее значение координаты осциллятора эволюционирует во времени по классическому закону затухающих гармонических колебаний, среднее значение импульса удовлетворяет уравнению Ланжевена, а дисперсия импульса - уравнению диффузии.

\section{СПИСОК ЦИТИРОВАННОЙ ЛИТЕРАТУРЫ}

[1] Davies E. B. Quantum Theory of Open Systems. London: Academic Press, 1976.

[2] Barchielli A., Belavkin V.P. Measurement continuous in time and a posteriori states in quantum mechanics // J. Phys. A. Math. Gen. 1991. V. 24. P. 1495-1514.

[3] Englert B. G., Morigi G. Five lectures on dissipative master equation // Lecture Notes in Phys. V. 611, 2002. P. 55-106; // E-print quant-ph/0206116, 2002.

[4] Ozawa M. Operations, disturbance, and simultaneous measurability // Phys. Rev. A. 2000. V. 62. P. 062101.

[5] Zurek W. H. Decoherence, einselection and the quantum origins of the classical // Rev. Mod. Phys. 2003. V. 75. P. 715; // E-print quant-ph/0105127, 2001.

[6] Isar A. Damped quantum harmonic oscillator: density operator and related quantities // E-print hep-th/9406142, 1994.

[7] Isar A. Uncertainty, entropy and decoherence of the damped harmonic oscillator in the Lindblad theory of open quantum systems // Fortschritte der Physik. 1999. V. 47. P. 855-879; // E-print quant-ph/9910019, 1999.

[8] Belavkin V.P. Nondemolition measurements, nonlinear filtering and dynamic programming of quantum stochastic processes // Lecture Notes in Control and Information. V. 121. Berlin: Springer, 1988. P. 245-265.

[9] Belavkin V.P. A stochastic posterior Schrödinger equation for counting nondemolition measurement // Lett. Math. Phys. 1990. V. 20. P. 85-89.

[10] Чуркин А. В. Точно решаемая задача для стохастического уравнения Шрёдингера в двумерном случае // Матем. заметки. 2001. Т. 69. № 5. С. 740-750.

Московский государственный университет им. М.В. Ломоносова

E-mail : churandr@mail.ru 